\title{
More efficient rejection of happy than of angry face distractors in visual search
}

\author{
GERNOT HORSTMANN, INGRID SCHARLAU, and ULRICH ANSORGE \\ Bielefeld University, Bielefeld, Germany
}

\begin{abstract}
In the present study, we examined whether the detection advantage for negative-face targets in crowds of positive-face distractors over positive-face targets in crowds of negative faces can be explained by differentially efficient distractor rejection. Search Condition A demonstrated more efficient distractor rejection with negative-face targets in positive-face crowds than vice versa. Search Condition B showed that target identity alone is not sufficient to account for this effect, because there was no difference in processing efficiency for positive- and negative-face targets within neutral crowds. Search Condition $\mathrm{C}$ showed differentially efficient processing with neutral-face targets among positive- or negative-face distractors. These results were obtained with both a within-participants (Experiment 1) and a between-participants (Experiment 2) design. The pattern of results is consistent with the assumption that efficient rejection of positive (more homogenous) distractors is an important determinant of performance in search among (face) distractors.
\end{abstract}

The possibility that affective stimulus characteristics, such as the valence of facial expressions of emotion, are preattentively available and can, thus, be used to guide attention has aroused the interest of researchers from often separate domains of research, such as perception (Eastwood, Smilek, \& Merikle, 2001; Nothdurft, 1993; Purcell, Stewart, \& Skov, 1996), social cognition (Hansen \& Hansen, 1988; Öhman, Lundqvist, \& Esteves, 2001), and emotion (Fox et al., 2000; Fox, Russo, \& Dutton, 2002; White, 1995). This threat advantage hypothesis is usually motivated by ecological or evolutionary considerations such as that it is adaptive to respond quickly and with little conscious preponderance to potentially damaging stimuli (cf. LeDoux, 1998). Therefore, the processing of negatively valenced social and nonsocial stimuli has priority over, for example, the processing of positive or beneficial stimuli. Under this assumption, the hypothesis that the negative valence of stimuli can be accessed preattentively has some plausibility.

An adequate means by which to test this hypothesis is the visual search paradigm, where the participant's task is to find a target among simultaneously presented distractors (e.g., Treisman \& Gelade, 1980; Wolfe, 1998). A target defined by a feature that is available to the cognitive system prior to an attentional scanning of distinct stimuli in the display can be found easily. In this case of efficient search, or perceptual pop-out, detection latency for the target is independent of the total number of stimuli

We are indebted to Christine Broermann and Gabriele-Anna Zantow for collecting the data, to Lily-Maria Silny for her assistance in manuscript preparation, and to Anne Hillstrom, as well as three anonymous reviewers, for their suggestions on prior versions of the present article. Address correspondence to G. Horstmann, Psychology Department, Bielefeld University, P.O. Box 100 131, D-33501 Bielefeld, Germany (e-mail: gernot.horstmann@uni-bielefeld.de). presented in a single display (set size). If, in contrast, the latency to find a target is positively related to set size, the detection of the target is probably the result of the serial deployment of attention to each stimulus in turn, until the target is detected. Search efficiency is defined as the slope $b$ of the linear equation $y=b x+a$, which relates the latency of finding the target $(y)$ to the set size $(x)$. In practice, a slope below $10 \mathrm{msec} / \mathrm{item}$ is often considered as shallow, and search is termed efficient. In contrast, a slope that exceeds $20 \mathrm{msec} / \mathrm{item}$ is considered as steep, and search is termed nonefficient (Wolfe, 1998).

One research strategy for demonstrating efficient processing of a particular feature in visual search is the search asymmetry design (e.g., Treisman \& Gormican, 1988; Treisman \& Souther, 1985; Wolfe, 2001). A search asymmetry amounts to the finding of pop-out and serial search with the use of only two types of stimuli, depending on which of the two serves as the target and which as the distractors. For example, searching for a $Q$ among Os is efficient, whereas searching for an $O$ among $Q$ s is not. Search asymmetries indicate that two stimuli can be discriminated by a single feature (e.g., Treisman \& Gormican, 1988; Treisman \& Souther, 1985) that is present in the pop-out stimulus, but less so in the serial search stimulus.

The hypothesis that facial threat is available preattentively predicts a search asymmetry, with (1) efficient search for a negative-face target among positive- or neutral-face distractors and (2) nonefficient search for a positive- or neutral-face target among negative-face distractors. Several studies have sought to confirm this prediction with schematic face stimuli (for a review, see Horstmann, in press). The results, however, have been inconclusive. Search for negative schematic faces has been mostly nonefficient. However, some studies have shown a relative search asymmetry (e.g., Fox et al., 2000; Horstmann, in press), with more efficient detection of angry 
targets among friendly distractors than vice versa (but see Nothdurft, 1993; Öhman et al., 2001; White, 1995).

Importantly, some of the evidence has indicated that the relative search asymmetry with affective faces might be due not solely to an efficient processing of negative-face targets, but also to a more efficient rejection of positive-face distractors. In particular, target-absent trials have shown an asymmetry too: Search slopes were shallower with crowds of positive-face distractors, relative to crowds of negativeface distractors (cf. Fox et al., 2000), suggesting that the participants rejected more positive-face distractors than negative-face distractors per unit of time (see also Hampton, Purcell, Bersine, Hansen, \& Hansen 1989; for a more general treatment of distractor rejection as a determinant of search efficiency, see Duncan \& Humphreys, 1989). Obviously, both target-present and target-absent responses can be facilitated with crowds of positive-face distractors.

The aim of the present study was to test this hypothesis of a more efficient rejection of positive-face distractors, with an emphasis on target-absent trials under conditions with similar amounts of target-distractor similarity. In Search Condition A, we demonstrated the basic observation of a better performance in target-absent trials when the target was a negative face and all the distractors were positive faces than vice versa. In Search Condition B, we tested whether the performance advantage for negativeface targets/positive-face distractors in Search Condition A was an effect of target identity. To that end, we used neutral face distractors. The neutral face stimulus was composed by superimposing the positive- and the negative-face stimuli (talking heads). If it is true that performance in Search Condition A was determined mainly by target face identity (e.g., Eastwood et al., 2001; Öhman et al., 2001) we should see virtually the same pattern of results as that in Search Condition A. However, if it is true that the differentially efficient rejection of the distractors determined performance, search efficiency should not differ for positive- and negative-face targets in Search Condition $\mathrm{B}$, in which only neutral distractors were used.

Finally, in Search Condition C, we tested for a more efficient rejection of the positive-face distractors without a negative-face target's being searched for. To that end, the participants searched for a neutral (talking head) face target among either negative-face or positive-face distractors. To the extent that performance is determined by distractor rejection, we expected to replicate the results of Search Condition A with a better performance in targetabsent trials consisting of positive-face distractors.

\section{EXPERIMENT 1}

\section{Method}

\section{Participants}

Twelve students ( 7 women) with a mean age of 25 years $(S D=$ $5.3)$ took part voluntarily and for pay (€6).

\section{Design *}

The complete experiment consisted of three search conditions, each comprising two blocks: In Search Condition A, the participants searched for a negative-face target among positive-face distractors or vice versa (cf. Treisman \& Souther, 1985); in Search Condition B, the participants searched for negative-face or positive-face targets among neutral talking head distractors; and in Search Condition C, the participants searched for talking head targets among negativeface or positive-face distractors. A constant-mapping procedure was used: Within each block, the identities of the target and the distractors were fixed. Each of the 6 conditions resulting from the orthogonal combination of the variables of set size ( 1 vs. $6 \mathrm{vs}$. 12) and target presence (target present vs. target absent) was presented 25 times. The variables of set size and target presence were varied randomly from trial to trial within blocks. Dependent variables were mean reaction times (RTs), mean percentages of errors, and the regression slopes and intercepts derived from relating the means to the set sizes. The order of the blocks within each search condition was balanced, as was the stimulus-response mapping (left vs. right response key for targetpresent vs. -absent responses). To keep the task simple for the participants, the stimulus-response mapping was held constant over the three search conditions. Each search condition (i.e., Search Conditions A, B, and $C$ ) appeared equally often at the first, second, or third serial position within the series, so that position effects were balanced.

\section{Apparatus}

The experiment was controlled by a computer, connected to a 15 -in. color monitor run with a resolution of $1,024 \times 768$ pixels for stimulus presentations and to a keyboard used to collect the manual responses. ERTS was used for event scheduling and response registration.

\section{Stimuli}

The faces measured $1.3 \times 1.3 \mathrm{~cm}$. Viewing distance was $120 \mathrm{~cm}$. In each trial, 1, 6, or 12 facial stimuli were presented without overlap inside an area of about $8.5 \times 6.5 \mathrm{~cm}$. Individual faces were presented on an imagined $4 \times 3$ (horizontal $\times$ vertical) position matrix. Mean distance between the faces (center to center) was $2.4 \mathrm{~cm}$. Average positions were altered by random displacement, separately computed for each position in each given trial. In particular, the average position of a stimulus was the center of a $3 \times 3$ grid, and the actual position of the stimulus was randomly chosen from the resulting nine positions. The distance of adjacent positions in the $3 \times 3$ grid was $2 \mathrm{~mm}$. The effect of this manipulation was a moderately irregular arrangement of the stimuli, intended to eliminate possible suprastimulus cues to the target that might result from a regular arrangement (Duncan \& Humphreys, 1989). The stimuli were presented in white on a black background.

\section{Procedure}

Written instructions were given prior to each condition. On request, the experimenter provided additional information. Each block was preceded by information about the identity of the target. For example, the participants were told that the targets in the following block would be happy faces and that they should search for the target and indicate with the correct response key whether it was present or absent. Instructions stressed both speed and accuracy. After this instruction, the participants worked on 20 practice trials, followed by 150 experimental trials.

Each trial began with a 1,000 -msec fixation cross, followed by the face stimuli. The stimuli remained on until a response was made, but a trial was aborted if no response was registered within $6 \mathrm{sec}$. If the participants pressed the wrong key, a 100 -msec tone provided error feedback. The intertrial interval was $1,100 \mathrm{msec}$.

\section{Results and Discussion}

\section{Data Treatment}

No anticipatory ( $<200 \mathrm{msec}$ ) or very long $(>5,000 \mathrm{msec})$ responses occurred. Errors occurred on $2.4 \%$ of the trials. For the RT analysis, mean correct RT for each experimental condition was calculated. The left column in Figure 1 depicts grand means. Panel A depicts the results for angry face targets among happy face distractors and for happy 
face targets among angry face distractors, panel B for angry face targets among talking head distractors and for happy face targets among talking head distractors, and panel $C$ for talking head targets among either happy face distractors or angry face distractors.

Because the predictions for all the conditions concerned the slopes of RT-set size functions, separate linear regressions with RT as the dependent variable and set size as the independent variable were computed for each participant. Thus, we obtained individual estimates of the two parameters $b$ (slope) and $a$ (intercept) of the linear regression equation $y=b x+a .^{1}$ For the present purposes, however, only the slopes are of interest. Analyses were also made on the slopes and the intercepts of the error scores (mean proportions of false responses). Table 1 shows the results of the ANOVAs; Table 2 shows the mean slopes and intercepts.

\section{Slopes}

Search Condition A. Searching for valenced targets among valenced distractors revealed a main effect for target presence, with RTs being shorter in target-present trials than in target-absent trials. The present-absent ratio was $1: 2.7$, which is in line with self-terminating serial search (i.e., about 1:2; cf. Treisman \& Gelade, 1980). There was also a main effect for target identity/distractor crowd identity, indicating more efficient search for negative targets among positive-face distractors than for positive targets among negative-face distractors. Planned comparisons using $t$ tests revealed that search was more efficient in both target-present and, importantly, target-absent trials $[\operatorname{ts}(11)>3.4, p s<.01$, two-tailed]. There was also a target (or crowd type) effect for the error slopes, which mirrors the corresponding RT effect and indicates that the crowd effect is not due to a speed-accuracy trade-off.

Search Condition B. Searching for the same targets among neutral faces revealed quite a different pattern of results: The only significant effect pertained to target presence, reflecting a present-absent ratio of the slopes of $1: 2.8$. The main effect for target was not significant. Planned comparisons using $t$ tests revealed no differences between slopes in positive-face, relative to negative-face, target conditions $[t \mathrm{~s}(11)<1]$.

Search Condition C. Searching for neutral faces among happy or angry faces revealed a main effect for target presence, reflecting a present-absent ratio of 1:2.5, and a main effect for crowd (or distractor type), reflecting more efficient search among positive-face distractors. The interaction between target presence and crowd identity was also significant, reflecting a smaller crowd effect in target-present trials $(6 \mathrm{msec})$ than in target-absent trials $(14 \mathrm{msec})$. The crowd effect (i.e., better performance with positive-face distractors) was significant in both targetpresent and target-absent trials $[t \mathrm{~s}(11)>3.6, p s<.01]$.

\section{EXPERIMENT 2}

Experiment 2 was a control experiment to test whether some of the effects of Experiment 1 were due to carryover effects between blocks. Thirty-six fresh participants (21 women; age: $M=28$ years, $S D=8.7)$ were tested, each in only one search condition.

\section{Results and Discussion}

The analyses corresponded to those in Experiment 1. The main results are reported in Figure 1, right column, and Tables 3 and 4.

The results closely corresponded to those of Experiment 1 , meaning that carryover effects between blocks were not responsible for the findings.

\section{GENERAL DISCUSSION}

The results are very clear. Search Condition A shows a relative search asymmetry, with more efficient search for negative-face targets among positive-face distractors and less efficient search for positive-face targets among negative-face distractors in target-present trials. As Eastwood et al. (2001) have pointed out, this result is difficult to interpret, because the effects of target identity and distractor identity are fully confounded.

Search Condition A also revealed search slope differences for the target-absent trials, meaning that distractor identity influenced performance: With no target being present, searching through positive-face distractors was more efficient than searching through negative-face distractors. However, it is possible that the identity of the target also had an effect on performance in the target-absent trials (see below).

Search Condition B revealed equally efficient search for positive-face and negative-face targets among affectively neutral face distractors (talking heads), meaning that target identity per se was not a sufficient condition for the differences in search efficiency that were found in Search Condition A. This result contrasts with some of Eastwood et al.'s (2001) findings: They found more efficient performance with negative-face than with positiveface targets among affectively neutral face distractors that were defined by a straight mouth line. This discrepancy indicates that the particular choice of the neutral-face baseline can be crucial (for a more general argument, see Jonides \& Mack, 1981, and below).

Finally, Search Condition C revealed more efficient performance with neutral (talking head) targets among positive-face distractors than with those among negativeface distractors. Because the target was the same in both blocks of Search Condition C, this effect must have been due to the different distractors. A striking feature of the present results is that the slopes for conditions of corresponding distractor identity in Search Conditions $\mathrm{C}$ and $\mathrm{A}$ were very similar; for example, search for a negative-face target among positive-face distractors in Experiment 1 was accomplished at a rate of $6 \mathrm{msec} / \mathrm{item}$, and search for a talking head target among positive-face distractors was carried out at a rate of $7 \mathrm{msec} / \mathrm{item}$. Taken together, the entire pattern of results suggests that the search asymmetry found in Search Condition A can be explained by differences in the speed by which the distractors were scanned. 

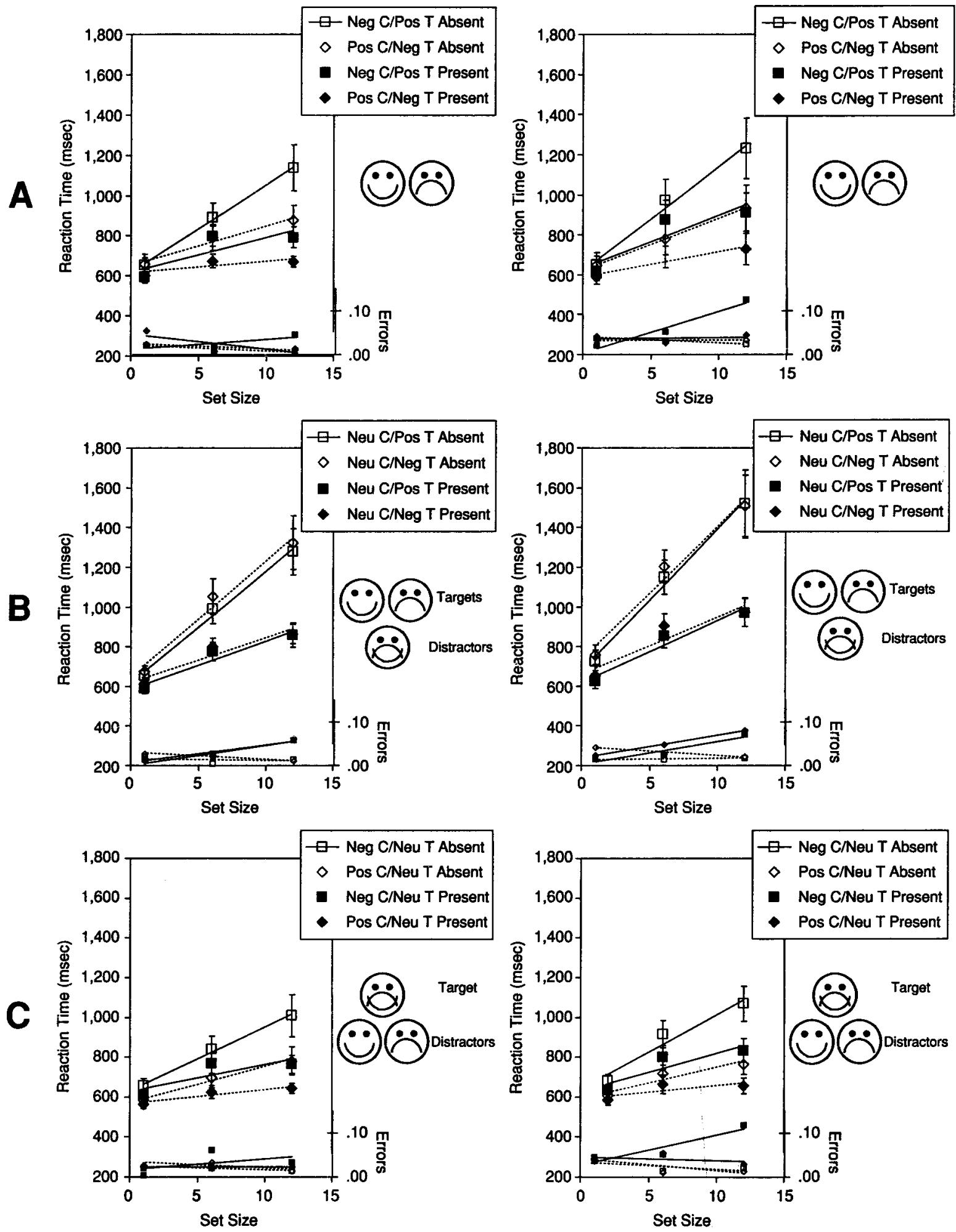

Figure 1. Mean correct reaction times and error rates for each of the 12 conditions in Search Conditions $A$ (top panel), B (middle panel), and C (bottom panel) in Experiments 1 (left column) and 2 (right column). Solid symbols represent targetpresent trials, and open symbols target-absent trials. Diamonds code for positive targets (Pos $T$ ) and/or negative crowds (Neg C), whereds squares code for negative targets (Neg T) and/or positive crowds (Pos C). The figure also displays the linear trends obtained by linear regression analysis. Error bars show the standard errors of the mean. 
Table 1

Summary of the $F$ Values From the ANOVAs on the Search Slopes and the Intercepts for the Reaction Time (RT) and the Error Data for Search Conditions A-C in Experiment 1

\begin{tabular}{|c|c|c|c|c|c|c|c|c|c|}
\hline \multirow{3}{*}{$\begin{array}{c}\text { Search } \\
\text { Condition }\end{array}$} & \multirow[b]{3}{*}{ Variable } & \multicolumn{4}{|c|}{ Slope } & \multicolumn{4}{|c|}{ Intercept } \\
\hline & & \multicolumn{2}{|c|}{ RT } & \multicolumn{2}{|c|}{ Errors } & \multicolumn{2}{|c|}{$\mathrm{RT}$} & \multicolumn{2}{|c|}{ Errors } \\
\hline & & $F$ & $M S_{e}$ & $F$ & $M S_{\mathrm{e}}$ & $F$ & $M S_{\mathrm{e}}$ & $F$ & $M S_{\mathrm{e}}$ \\
\hline \multirow[t]{3}{*}{ A } & Presence (1) & 14.79 & 327.2 & 0.00 & $1.36 \mathrm{E}-05$ & 0.74 & $6,281.5$ & 0.55 & $1.66 \mathrm{E}-03$ \\
\hline & Crowd (2) & 16.47 & 229.8 & 11.00 & $6.82 \mathrm{E}-06$ & 0.25 & $8,518.0$ & 2.67 & $1.58 \mathrm{E}-03$ \\
\hline & (1) $\times(2)$ & 4.42 & 110.3 & 3.14 & $1.06 \mathrm{E}-05$ & 2.35 & $2,101.5$ & 3.48 & 7.34E-04 \\
\hline \multirow[t]{3}{*}{ B } & Presence (1) & 15.60 & $1,013.6$ & 4.12 & $6.12 \mathrm{E}-05$ & 1.69 & $4,359.1$ & 7.32 & $6.29 \mathrm{E}-04$ \\
\hline & Crowd (2) & 0.04 & 412.2 & 0.07 & $2.94 \mathrm{E}-05$ & 4.45 & $2,044.9$ & 0.12 & $1.33 \mathrm{E}-03$ \\
\hline & (1) $\times(2)$ & 1.14 & 108.6 & 0.07 & $2.94 \mathrm{E}-05$ & 0.28 & $1,011.6$ & 1.26 & $8.75 \mathrm{E}-04$ \\
\hline \multirow[t]{3}{*}{ C } & Presence (1) & 8.35 & 295.4 & 4.12 & $1.59 \mathrm{E}-05$ & 0.90 & 878.5 & 0.65 & $1.15 \mathrm{E}-03$ \\
\hline & Crowd (2) & 26.23 & 47.2 & 1.69 & $1.97 \mathrm{E}-05$ & 16.75 & $2,757.0$ & 0.59 & $1.86 \mathrm{E}-03$ \\
\hline & (1) $\times(2)$ & 12.42 & 16.3 & 0.00 & $1.36 \mathrm{E}-05$ & 0.63 & 350.8 & 0.05 & 2.08E-03 \\
\hline
\end{tabular}

Note-For all $F \mathrm{~s}, d f \mathrm{~s}=1,11$. Italicized values exceed the critical $F=4.84, p=.05$.

The entire pattern of results does not favor the hypothesis that threatening or negative faces are processed more efficiently than positive faces, because the obtained differences in target-present trials can be accounted for by a less efficient scanning through a negative-face crowd than through a positive-face crowd. One might argue that this conclusion is unwarranted because the present experiments presented cartoon-like icons with little resemblance to real faces (cf. Horstmann, Borgstedt, \& Heumann, 2006). Thus, it might be questioned whether the threat advantage hypothesis, which is ultimately concerned with real faces, makes any predictions for the simplified face stimuli or whether this hypothesis can be tested only with reproductions of real faces (Horstmann \& Bauland, 2006). Previous research, however, has taken for granted that strongly simplified schematic facial stimuli are relevant for the threat advantage hypothesis (e.g., Eastwood et al., 2001; Fox et al., 2000; Öhman et al., 2001; White, 1995). ${ }^{2}$

What is the mechanism responsible for the difference in search efficiency between positive- and negative-face distractor conditions? We will consider two types of explanations: one emotional and one perceptual. To start with the emotional accounts, Fox et al. (2000) assumed that searching through a crowd of negative faces is relatively slow because the negative faces activate a threat detection module, which, in turn, slows down the speed of the attentional disengagement from the threatening stimuli (see Eastwood, Smilek, \& Merikle, 2003, for a similar account). Alternatively, negatively valenced stimuli may induce a general slowing of the search processes by inducing general interference (cf. Yiend \& Mathews, 2001) proportional to the number of negative stimuli. ${ }^{3}$ One might argue that the latter account would predict less efficient search for negative-face targets than for positive-face targets among neutral-face distractors in Search Condition B. However, with only one negative-face stimulus being present, low experimental power might have masked the effect. Finally, Leppänen and Hietanen (2003) provided evidence that happy faces are categorized more quickly than angry faces and that this effect is partly genuinely emotional.

It is also possible to give a perceptual account of the relatively efficient rejection of happy face distractors. First, positive faces may be perceptually more easy to process than negative faces and, thus, rejected more efficiently as distractors. At least intuitively, positive faces are simpler than negative faces, in that positive mouths repeat the outline of the spatially adjacent lower part of the face, whereas the curvature of the negative mouth differs from the adjacent outline of the lower part of the face. If one takes into consideration perceptual grouping by similarity and proximity in addition, the components of the happy face would be expected to merge more easily into a good gestalt than would the angry face distractors.

Second, the number of distractors that can be simultaneously rejected as a group (Treisman \& Souther, 1985) might be larger with positive-face than with negative-face distractors, decreasing search efficiency with the latter. Duncan and Humphreys (1989) argued that high distractordistractor similarity supports efficient distractor rejection. Once again, positive-face distractors, being self-similar in

Table 2

Summary of the Search Slopes and the Intercepts for the Reaction Time (RT) and the Error Data for Search Conditions $A-C$ in Experiment 1

\begin{tabular}{|c|c|c|c|c|}
\hline \multirow[b]{2}{*}{ Condition } & \multicolumn{2}{|c|}{ Slope } & \multicolumn{2}{|c|}{ Intercept } \\
\hline & RT & Errors & RT & Errors \\
\hline \multicolumn{5}{|c|}{ Search Condition A } \\
\hline Pos T/Neg C present & 17.3 & .002 & 618 & .014 \\
\hline Neg T/Pos C present & 6.0 & -.003 & 611 & .048 \\
\hline Pos T/Neg C absent & 43.8 & .000 & 617 & .020 \\
\hline Neg T/Pos C absent & 19.7 & -.001 & 651 & .024 \\
\hline \multicolumn{5}{|c|}{ Search Condition B } \\
\hline Pos T/Neu C present & 24.3 & .004 & 586 & .007 \\
\hline Neg T/Neu C present & 22.2 & .004 & 618 & .001 \\
\hline Pos T/Neu C absent & 57.4 & .000 & 616 & .017 \\
\hline Neg T/Neu C absent & 61.8 & -.001 & 638 & .030 \\
\hline \multicolumn{5}{|c|}{ Search Condition C } \\
\hline Neu T/Neg C present & 13.4 & .002 & 630 & .015 \\
\hline Neu T/Pos C present & 7.3 & .000 & 563 & .022 \\
\hline Neu T/Neg C absent & 31.8 & -.001 & 634 & .020 \\
\hline Neu T/Pos C absent & 17.5 & -.003 & 576 & .033 \\
\hline
\end{tabular}

Note-Pos, positive; Neg, negative; Neu, neutral (= talking head); $T$, target; $\mathrm{C}$, crowd. 
Table 3

Summary of the $F$ Values From the ANOVAs on the Search Slopes and the Intercepts for the Reaction Time (RT) and the Error Data for Search Conditions A-C in Experiment 2

\begin{tabular}{|c|c|c|c|c|c|c|c|c|c|}
\hline \multirow{3}{*}{$\begin{array}{c}\text { Search } \\
\text { Condition }\end{array}$} & \multirow[b]{3}{*}{ Variable } & \multicolumn{4}{|c|}{ Slope } & \multicolumn{4}{|c|}{ Intercept } \\
\hline & & \multicolumn{2}{|c|}{ RT } & \multicolumn{2}{|c|}{ Errors } & \multicolumn{2}{|c|}{ RT } & \multicolumn{2}{|c|}{ Errors } \\
\hline & & $F$ & $M S_{e}$ & $F$ & $M S_{\mathrm{e}}$ & $F$ & $M S_{e}$ & $F$ & $M S_{\mathrm{e}}$ \\
\hline \multirow[t]{3}{*}{ A } & Presence (1) & 20.0 & 237.7 & 4.5 & $5.66 \mathrm{E}-05$ & 0.3 & $1,872.2$ & 2.7 & $9.86 \mathrm{E}-04$ \\
\hline & Crowd (2) & 19.1 & 252.7 & 6.1 & $2.78 \mathrm{E}-05$ & 0.4 & $17,334.4$ & 1.4 & $1.33 \mathrm{E}-03$ \\
\hline & (1) $\times(2)$ & 4.0 & 115.7 & 17.4 & $2.03 E-05$ & 4.0 & $1,615.3$ & 4.9 & 8.95E-04 \\
\hline \multirow[t]{3}{*}{ B } & Presence (1) & 12.6 & $1,500.8$ & 11.3 & $4.15 \mathrm{E}-05$ & 4.0 & $11,892.4$ & 2.4 & $1.39 \mathrm{E}-03$ \\
\hline & Crowd (2) & 0.5 & 266.9 & 0.1 & $2.94 \mathrm{E}-05$ & 3.8 & $6,833.3$ & 6.0 & $1.01 \mathrm{E}-03$ \\
\hline & (1) $\times(2)$ & 0.1 & 50.1 & 1.0 & $1.88 \mathrm{E}-05$ & 0.2 & 886.8 & 0.7 & $7.47 \mathrm{E}-04$ \\
\hline \multirow[t]{3}{*}{ C } & Presence (1) & 15.8 & 120.9 & 1.9 & $1.41 \mathrm{E}-04$ & 2.1 & $1,966.0$ & 0.0 & $2.54 \mathrm{E}-03$ \\
\hline & Crowd (2) & 19.0 & 158.2 & 9.8 & $3.18 \mathrm{E}-05$ & 9.4 & $4,008.6$ & 1.4 & $1.35 \mathrm{E}-03$ \\
\hline & (1) $\times(2)$ & 8.3 & 29.8 & 1.0 & $2.50 \mathrm{E}-05$ & 3.0 & 542.4 & 0.3 & $2.08 \mathrm{E}-03$ \\
\hline
\end{tabular}

Note-For all $F \mathrm{~S}, d f \mathrm{~s}=1,11$. Italicized values exceed the critical $F=4.84, p=.05$.

that the components (eyes and mouth) of their facial expressions are similar to adjacent face contours, would lead to more efficient search than would the more complex perceptual organization of negative-face distractors.

A third factor influencing the efficiency of distractor rejection is target-distractor similarity (Duncan \& Humphreys, 1989). To illustrate, in searching for an $O$ versus an $\mathrm{F}$ among Es, there is presumably a search asymmetry favoring the $\mathrm{O}$, because the $\mathrm{O}$-target is more dissimilar to the E-distractor than is the F-target. Importantly, no such effect was apparently active in the present study. In Search Condition B, performance with neutral-face distractors was the same for blocks with positive-face targets and blocks with negative-face targets. However, this factor might help one to understand the differences between the present study and that of Eastwood et al. (2001). Eastwood et al. (2001) used straight-line distractors and presented them with either the positive-face target or the negativeface target within a single block (with target identity varying randomly from trial to trial). Importantly, however, Eastwood et al. (2001) did not test for different amounts of perceived target-distractor similarity with their neutralface distractors under different target conditions. Therefore, different target-distractor similarities might have been a factor in that study. For example, assuming that target-distractor similarity was higher with positive- than with negative-face targets in that study, there might have been more rescanning of the distractors, in cases in which the target was not quickly found, with the positive-face than with the negative-face targets.

Of course, this "explanation" is post hoc and speculative. The important point is that it highlights a major methodological requirement not met by the study of Eastwood et al. (2001) but met by the present research: Differences in the ease of distractor rejection caused by different amounts of perceived target-distractor similarity must be ruled out. Adequate control conditions are constantmapping procedures, where the identities of the target and the distractors are constant within a block. Evidence for same target-distractor similarity is obtained if distractor rejection (i.e., search efficiency) in the target-absent trials is not influenced by target identity in a given block.
In other words, if we seek to identify differences in the processing of emotional content in visual search tasks, we have to rule out the possibility that results can be equally well explained by mere perceptual differences between different emotional conditions.

We wish to conclude with three suggestions. First, we suggest that more attention should be paid to target-absent trials in visual search tasks, because these trials inform about distractor rejection efficiency, which is an often overlooked determinant of overall search efficiency. Second, the question of whether search efficiency depends on characteristics of the target or the distractor is probably an oversimplification, given that search performance depends on the similarity relations between target and distractors (Duncan \& Humphreys, 1989). For this reason, neutral distractors have to be chosen with special care. And third, we want to argue that target-absent trials in constantmapping procedures (i.e., ones in which the identities of

Table 4

Summary of the Search Slopes and the Intercepts for the Reaction Time (RT) and Error Data for Search Conditions A-C in Experiment 2

\begin{tabular}{lrrrrr}
\hline & \multicolumn{2}{c}{ Slope } & & \multicolumn{2}{c}{ Intercept } \\
\cline { 2 - 3 } \cline { 5 - 6 } & \multicolumn{1}{c}{ RT } & Errors & & RT & Errors \\
& \multicolumn{1}{c}{ Search Condition A } & & \\
Pos T/Neg C present & 26.3 & .009 & 635.1 & .004 \\
Neg T/Pos C present & 12.4 & .000 & & 589.4 & .036 \\
Pos T/Neg C absent & 52.4 & -.001 & 618.9 & .038 \\
Neg T/Pos C absent & 26.1 & .001 & 619.4 & .032 \\
& & & & \\
Pos T/Neu C present & 31.2 & .005 & 618.5 & .002 \\
Neg T/Neu C present & 28.6 & .006 & 661.3 & .018 \\
Pos T/Neu C absent & 71.7 & .000 & 677.9 & .012 \\
Neg T/Neu C absent & 67.7 & -.002 & 728.1 & .041 \\
& Search Condition C & & \\
Neu T/Neg C present & 17.6 & .008 & 644.7 & .026 \\
Neu T/Pos C present & 6.3 & .000 & 594.0 & .045 \\
Neu T/Neg C absent & 34.8 & .000 & 668.9 & .034 \\
Neu T/Pos C absent & 14.4 & -.003 & 605.0 & .040 \\
\hline Note
\end{tabular}

Note-Pos, positive; Neg, negative; Neu, neutral (= talking head); target; $\mathrm{C}$, crowd. 
the target and the distractors are held constant) should be used as a diagnostic for equal target-distractor similarities between two targets and a neutral distractor.

\section{REFERENCES}

DUNCAN, J., \& HumphreYs, G. W. (1989). Visual search and stimulus similarity. Psychological Review, 96, 433-458.

EAstwooD, J. D., SmILE, D., \& MERIKLE, P. M. (2001). Differential attentional guidance by unattended faces expressing positive and negative emotion. Perception \& Psychophysics, 63, 1004-1013.

EAsTwOOD, J. D., SMLEK, D., \& MERIKLE, P. M. (2003). Negative facial expression captures attention and disrupts performance. Perception \& Psychophysics, 65, 352-358.

Fox, E., Lester, V., Russo, R., Bowles, R. J., Pichler, A., \& Durron, K. (2000). Facial expressions of emotion: Are angry faces detected more efficiently? Cognition \& Emotion, 14, 61-92.

Fox, E., Russo, R., \& DUTroN, K. (2002). Attentional bias for threat: Evidence for delayed disengagement from emotional faces. Cognition \& Emotion, 16, 355-379.

hampton, C., Purcell, D. G., Bersine, L., Hansen, C. H., \& HaNSEN, R. D. (1989). Probing "pop-out": Another look at the face-in-thecrowd effect. Bulletin of the Psychonomic Society, 27, 563-566.

HANSEN, C. H., \& HANSEN, R. D. (1988). Finding the face in the crowd: An anger superiority effect. Journal of Personality \& Social Psychology, 54, 917-924.

HoRstManN, G. (in press). Preattentive face processing: What do visual search experiments with schematic faces tell us? Visual Cognition.

HoRSTMANN, G., \& BAULAND, A. (2006). Search asymmetries with real faces: Testing the anger-superiority effect. Emotion, 6, 193-207.

Horstmann, G., Borgstedt, K., \& Heumann, M. (2006). Flanker effects with faces may depend on perceptual as well as emotional differences. Emotion, 6, 28-39.

JoNIDES, J., \& MACK, R. (1984). On the cost and benefit of cost and benefit. Psychological Bulletin, 96, 29-44.

LEDoux, J. E. (1998). The emotional brain: The mysterious underpinnings of emotional life. London: Phoenix.

LEPPÄNEN, J. M., \& HIETANEN, J. K. (2003). Affect and face perception: Odors modulate the recognition advantage of happy faces. Emotion, 3, 315-326.

LunDQvist, D., Esteves, F., \& ÖHMAN, A. (1999). The face of wrath: Critical features for conveying facial threat. Cognition \& Emotion, 13, 691-711.

NotHDURFT, H.-C. (1993). Faces and facial expressions do not pop out. Perception, 22, 1287-1298.

Öhman, A., Lundevist, D., \& Esteves, F. (2001). The face in the crowd revisited: A threat advantage with schematic stimuli. Journal of Personality \& Social Psychology, 80, 381-396.
Purcell, D. G., Stewart, A. L., \& Skov, R. B. (1996). It takes a confounded face to pop out of a crowd. Perception, 25, 1091-1 108.

TREISMAN, A. M., \& GELADE, G. (1980). A feature-integration theory of attention. Cognitive Psychology, 12, 97-136.

Treisman, A. [M.], \& Gormican, S. (1988). Feature analysis in early vision: Evidence from search asymmetries. Psychological Review, 95, $15-48$.

Treisman, A. [M.], \& Souther, J. (1985). Search asymmetry: A diagnostic for preattentive processing of separable features. Journal of Experimental Psychology: General, 114, 285-310.

WHITE, M. (1995). Preattentive analysis of facial expressions of emotion. Cognition \& Emotion, 9, 439-460.

WolfE, J. M. (1998). Visual search. In H. Pashler (Ed.), Attention (pp. 13-73). London: University College London Press.

WOLFE, J. M. (2001). Asymmetries in visual search: An introduction. Perception \& Psychophysics, 63, 381-389.

YIEND, J., \& MATHEWs, A. (2001). Anxiety and attention to threatening pictures. Quarterly Journal of Experimental Psychology, 54A, 665-681.

\section{NOTES}

1. The function relating RT to set size has a nonlinear component, which is not modeled by the linear equation. We considered using only the set sizes 6 and 12 to estimate slopes; set size 1 is qualitatively different, because only one stimulus is presented. However, a control for Search Condition C, in which set size 1 was replaced by set size 2 , rendered practically the same results as those for Search Condition $\mathrm{C}$ (the slopes were Neu T/Neg C Present, $14 \mathrm{msec} /$ item; Neu T/Pos C Present, $5 \mathrm{msec} / \mathrm{item}$; Neu T/Neg C Absent, $37 \mathrm{msec} / \mathrm{item}$; Neu T/Pos C Absent, $12 \mathrm{msec} / \mathrm{item})$. Apparently, the qualitative difference from set size 1 to set size $1+n$ does not explain the nonlinearity. Possibly, the nonlinearity comes into play as the display gets increasingly crowded, allowing for more perceptual interactions between neighboring stimuli. However, because it is unclear where the exact locus of the nonlinearity is, all three set sizes are used for the estimation of slope.

2. Some researchers have tested facial threat for schematic stimuli with ratings (e.g., Lundqvist, Esteves, \& Öhman, 1999) and have found V-shaped eyebrows to be a critical feature. The importance of eyebrows, however, has been contested: Fox et al. (2000) argued that an evolved threat detector, to subserve its function, should respond to ambiguousthreat/negative-valence stimuli (i.e., browless negative stimuli). A more general point is that the ratings still pertained to schematic facial stimuli, with unknown relevance for real faces.

3. This possibility was actually suggested to us by an anonymous reviewer.

(Manuscript received April 26, 2005;

revision accepted for publication April 10, 2006.) 